\title{
Cetuximab in preoperative treatment of rectal cancer - term outcome of the XERT trial
}

\author{
Vaneja Velenik ${ }^{1}$, Janja Ocvirk², Irena Oblak ${ }^{1}$, Franc Anderluh ${ }^{1}$ \\ ${ }^{1}$ Department of Radiotherapy, Institute of Oncology Ljubljana, Ljubljana, Slovenia \\ 2 Department of Medical Oncology, Institute of Oncology Ljubljana, Ljubljana, Slovenia
}

Radiol Oncol 2012; 46(3): 252-257.

Received 10 February 2012

Accepted 19 March 2012

Correspondence to: Assist. Prof Vaneja Velenik, MD, PhD, Institute of Oncology Ljubljana, Zaloska 2, 1000 Ljubljana, Slovenia. Phone: +38615879661; Fax: +38615879400; E-mail: vvelenik@onko-i.si

Disclosure: No potential conflicts of interest were disclosed.

Background. Preoperative capecitabine-based chemoradiotherapy (CRT) is feasible for the treatment of resectable locally advanced rectal cancer (LARC). To try to improve efficacy, we conducted a phase II study in which the epidermal growth factor receptor-targeting monoclonal antibody cetuximab was added to capecitabine-based CRT. The results for long-term survival and for an analysis investigating the relationship between survival and patient and disease characteristics, including tumour KRAS mutation status, and surgery type, are presented.

Patients and methods. Patients with resectable LARC received capecitabine $\left(1250 \mathrm{mg} / \mathrm{m}^{2}\right.$ twice daily, orally) for 2 weeks followed by cetuximab alone ( $400 \mathrm{mg} / \mathrm{m}^{2}$ for 1 week) and then with CRT ( $250 \mathrm{mg} / \mathrm{m}^{2} /$ week) comprising capecitabine ( $825 \mathrm{mg} / \mathrm{m}^{2}$ twice daily) and radiotherapy to the small pelvis (45 Gy in 25 1.8-Gy fractions), five days a week for five weeks. Surgery was conducted six weeks following CRT, with post-operative chemotherapy with capecitabine ( $1250 \mathrm{mg} / \mathrm{m}^{2}$ twice daily for 14 days every 21 days) three weeks later.

Results. Forty-seven patients were enrolled and 37 underwent treatment. Twenty-eight of the patients (75.7\%) had T3N+ disease. Thirty-six patients were evaluable for efficacy. The median follow-up time was 39.0 months (range 5.0-87.0). The three-year local control, disease-free survival, relapse-free survival and overall survival rates were $96.9 \%(95 \%$ Cl 90.0--100), 72.2\% (57.5--86.9), 74.3\% (95\% Cl 59.8--88.8) and 68.1\% (95\% Cl 36.7--99.4), respectively. There was no significant association between survival and gender, age, tumour location in the rectum, type of surgery, pathological T or $\mathrm{N}$ status, tumour regression grade or tumour KRAS mutation status, although sample sizes were small.

Conclusions. Preoperative cetuximab plus capecitabine-based CRT was feasible in patients with resectable LARC and was associated with an impressive three-year local control rate. The use of tumour KRAS mutation status as a biomarker for the efficacy of cetuximab-based regimens in this setting requires further investigation.

Key words: rectal cancer; radio-chemotherapy; cetuximab

\section{Introduction}

Preoperative concurrent chemoradiotherapy (CRT) is associated with improved local tumour control compared with preoperative radiotherapy ${ }^{1,2}$ and is a commonly used approach for the treatment of stage II/III locally advanced rectal cancer (LARC). Since the initial observation of the clinical advantages of CRT over radiotherapy, methods of further improving outcomes have been investigated.

In a previous phase II study, we reported that the use of preoperative capecitabine-based CRT was feasible for the treatment of resectable LARC. ${ }^{3}$ We then attempted to improve the efficacy of this regimen by adding the epidermal growth factor receptor (EGFR)-targeting monoclonal antibody, cetuximab. The rationale for the use of this combination was based on the radiosensitizing effects observed with cetuximab in in vivo model systems ${ }^{4,5}$, supported by clinical evidence from a randomized phase III trial investigating the combination of cetuximab and radiotherapy in the treatment of locally advanced squamous cell carcinoma of the head and neck. ${ }^{6,7}$ In the phase III trial, the combination of 
cetuximab and radiotherapy was significantly more beneficial, in terms of both locoregional control and survival, than radiotherapy alone.

In 2007, we designed a prospective, non-randomized, open label phase II study to investigate the impact of adding cetuximab to preoperative capecitabine-based CRT for the treatment of 37 patients with resectable LARC. The primary endpoint of the trial was pathological complete response (pCR). Results reported in 2010 showed a pCR of $8 \%$ (3 patients) with overall-, $\mathrm{T}$ - and $\mathrm{N}$-downstaging rates of $73 \%, 57 \%$ and $81 \%$, respectively. The total sphincter preservation rate was $76 \%{ }^{8}$

The identification of biomarkers to tailor treatment to patients most likely to benefit has become an integral part of the investigation of novel treatments and regimens. ${ }^{9,10}$ Retrospective analyses of data from randomized trials demonstrated significant improvements in survival when cetuximab was added to standard chemotherapy regimens for the treatment of patients with metastatic CRC not harbouring KRAS mutations. ${ }^{11,12}$ While the reported incidence rates of tumour KRAS mutations in rectal cancer are lower than those for CRC, with rates ranging between $13 \%$ and $48 \%{ }^{13-17}$ compared with the $55-70 \%$ reported for metastatic colorectal cancer (mCRC), the presence of such mutations may still have a significant impact on treatment outcome.

We report here the results of the long-term follow-up of our phase II study ${ }^{8}$, with three-year survival results, together with findings from an analysis conducted to investigate any relationship between survival and baseline patient and disease characteristics, including tumour KRAS mutation status, and the type of surgery conducted.

\section{Patients and methods}

Details of the study design, eligibility criteria, treatment and assessments have been reported in detail previously. ${ }^{8}$ The study was approved by the relevant ethics committees and was conducted in accordance with the Declaration of Helsinki. It was registered at ClinicalTrials.gov (NCT00689702). All patients provided written informed consent.

\section{Patients and study design}

Briefly, patients with histologically-confirmed International Union Against Cancer (UICC) stage II/III adenocarcinoma of the rectum and a World Health Organization (WHO) performance status (PS) of $\leq 2$ and who had not previously received ra- diotherapy and/or chemotherapy for their disease were included in the study. ${ }^{8}$ The extent of locoregional disease was determined by magnetic resonance imaging (MRI).

Patients were scheduled to receive eight weeks of treatment, at the end of which the primary tumour was re-evaluated with pelvic MRI and response evaluated according to Response Evaluation Criteria In Solid Tumours (RECIST). Definitive surgery was scheduled to take place four to six weeks after the completion of CRT. A decision on the type of surgery to be conducted was taken prior to the start of preoperative CRT. Three cycles of postoperative chemotherapy, each lasting three weeks, were recommended to start within six weeks of surgery. After the operation the post-treatment surveillance was regularly done. ${ }^{18}$

\section{Treatment}

Capecitabine $\left(1250 \mathrm{mg} / \mathrm{m}^{2}\right.$ twice daily orally) was administered for two weeks, followed, on day 1 of week 3 , by cetuximab IV $\left(400 \mathrm{mg} / \mathrm{m}^{2}\right)$. Starting on day 1 of week 4, patients received cetuximab $\left(250 \mathrm{mg} / \mathrm{m}^{2} /\right.$ week) plus CRT with capecitabine $\left(825 \mathrm{mg} / \mathrm{m}^{2}\right.$ twice daily) and radiotherapy to the small pelvis (45 Gy in 25 1.8-Gy fractions), five days a week for five weeks. Radiation was delivered using three-dimensional conformal computed tomography (CT)-based treatment planning. The chemotherapy component of CRT was initiated on the first day and finished on the last day of radiotherapy (including weekends). Surgery was to be sphincter-sparing where possible. Post-operative chemotherapy comprised capecitabine $\left(1250 \mathrm{mg} / \mathrm{m}^{2}\right.$ twice daily for two weeks every three weeks days).

\section{Assessments}

During treatment patients were monitored weekly for safety. Tumour response was assessed five weeks after the end of preoperative treatment. After surgery, histological examination of excised tissue was performed to determine the degree of pathological tumour response. Histological regression of the primary tumour was evaluated using a previously described tumour regression grading (TRG) system: TRG $0=$ no regression, TRG $1=$ minimal regression, TRG $2=$ moderate regression, TRG $3=$ good regression and TRG $4=$ total regression. ${ }^{19,20}$

\section{Tumour KRAS mutation analysis}

Tumour tissue for KRAS analysis was obtained prior to neoadjuvant treatment and at surgery. After 
microdissection, intratumoural gene expression levels and analysis of seven KRAS mutations (codon 12 and codon 13) was carried out using a proprietary procedure, the ResponseDX: Colon ${ }^{\mathrm{TM}}$ system (Response Genetics Inc., Los Angeles, California, USA) referred to in previous publications..$^{21,22}$

\section{Statistical methods and considerations}

The primary endpoint of the study was the pathological complete response (pCR) rate (defined as the complete disappearance of all tumour cells in surgically excised tissue), results for which have been reported in a separate publication. ${ }^{8}$ Secondary endpoints reported here are: locoregional control (defined as the time from inclusion to documentation of tumour recurrence in the pelvis. It is emphasized that the development, or progression, of metastatic disease did not constitute local failure), relapse-free survival (defined as the time from inclusion to the first occurrence of disease relapse [local or distant], death or date of last follow-up), disease-free survival (defined as the time to relapse, second cancer, or death from any cause, whichever came first) and overall survival (defined as time from inclusion to the date of death from any cause or to the date of last follow-up).

The Kaplan-Meier method was used to estimate the rates of overall, relapse-free, disease-free and local relapse-free survival. A univariate analysis was performed to investigate any association between relapse-free and disease-free survival and parameters including gender, age, tumour location in the rectum (low, middle, upper third), type of surgery (abdominoperineal amputation, sphincter sparing), pathological T or N status, TRG and tumour KRAS mutation status. The log-rank test was used to test the significance between the subgroups for this endpoint. The cumulative incidence approach was used to estimate the rates for disease-specific mortality, local recurrence and distant metastasis. Statistical analysis was performed using the SPSS statistical software package, version 12 (SPSS Inc., Chicago, IL, USA).

\section{Results}

\section{Patients}

Between February 2007 and September 2008, 43 patients were recruited into the study. Thirty-seven of these patients subsequently received preoperative treatment with cetuximab plus capecitabinebased CRT. Median age was 55 years, $81.1 \%$ were males, and $90.9 \%$ presented with stage III disease. The median distance of tumour from the anal verge was $6.0 \mathrm{~cm}$ (range 1.0-11.0 cm).

\section{Preoperative treatment, surgery and postoperative chemotherapy}

Cetuximab administration was discontinued after the first dose in four patients due to hypersensitivity reactions: these patients continued to receive capecitabine and radiotherapy. All 37 patients received the planned dose of radiation. Only two patients $(5.4 \%)$ received less than $90 \%$ of the planned dose of capecitabine, one due to grade 3 hepatotoxicity and one due to grade 3 diarrhoea.

All 37 patients underwent surgery. Sphinctersparing surgery was performed in 26 patients (70.3\%) and abdominoperineal amputation in 11 patients $(29.7 \%)$. One patient experienced back pain a few months after surgery and, on re-examination of the pretreatment MRI scan, was found to have had sacral bone metastasis prior to treatment. This patient was excluded from the survival and KRAS analyses. Tumour tissue from 32 patients was available for KRAS analysis and KRAS mutations were detected in the tumour tissue of 13 patients $(40.6 \%)$.

Postoperative chemotherapy was administered to 34 patients. Thirty-two were able to receive all three planned cycles at the recommended dose: among these patients, one developed grade 3 leukopenia and one had grade 2 vomiting. The remaining two patients received two cycles of capecitabine due to the development of grade 2 diarrhoea.

\section{Treatment outcome}

The median follow-up time for all 36 patients was 39.0 months (range 5.0-87.0 months). The median time to disease recurrence was 35.0 months (range 5.0-87.0 months). Local failure occurred in one patient $(2.8 \%), 11.5$ months after the end of treatment. The three-year local control rate was $96.9 \%$ (95\% CI 90.9-100). Disease dissemination was observed in nine patients $(25.0 \%)$ with the following pattern of distribution: liver $(n=3 ; 8.3 \%)$, lung $(n=3$; $8.3 \%)$, suprarenal gland $(n=1 ; 2.8 \%)$, bones $(n=1$; $2.8 \%$ ) and synchronous lung plus retroperitoneum $(n=1 ; 2.8 \%)$. The latest distant failure was observed 26 months after the end of treatment. No secondary malignancies were observed. The three-year relapse-free survival rate was $74.3 \%$ (95\% CI 59.888.8) (Figure 1) and the three-year disease-free survival rate was $72.2 \%$ (95\% CI 57.5-86.9) (Figure 2). 


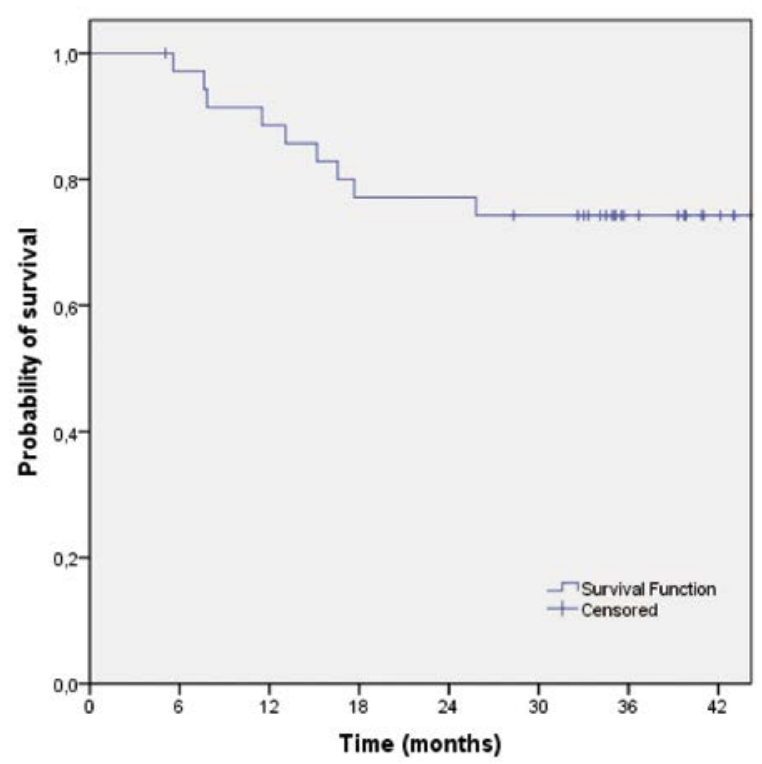

FIGURE 1. Relapse-free survival of patients treated with preoperative capecitabine-base chemotherapy and cetuximab $(n=36)$.

The median survival has not yet been reached. The three-year survival rate was $68.1 \%$ (95\% CI 36.7-99.4) (Figure 3).

There was no significant association between relapse- or disease-free survival and gender, age, tumour location in the rectum, type of surgery, pathological $\mathrm{T}$ or $\mathrm{N}$ status, TRG or tumour KRAS mutation status (Table 1).

\section{Deaths}

As of September 2011, six of the 36 patients had died due to the following causes: rectal cancer $(n=4)$, preoperative complications $(n=1)$ and nondisease or treatment-related cause (accident at work) $(\mathrm{n}=1)$.

\section{Discussion}

The results of this trial demonstrated that the addition of cetuximab to preoperative capecitabinebased CRT led to a pCR rate of $8 \%{ }^{8}$ with long-term results showing a three-year local control rate of $96.9 \%$, a three-year disease-free survival rate of $72.2 \%$ and a three-year overall survival rate of $68.1 \%$. The treatment regimen was well tolerated.

Since the results of the landmark randomized trial reported by the German Rectal Cancer Study Group in 2004, which confirmed the local control and reduced toxicity benefits of preoperative fluorouracil-based CRT over post-operative

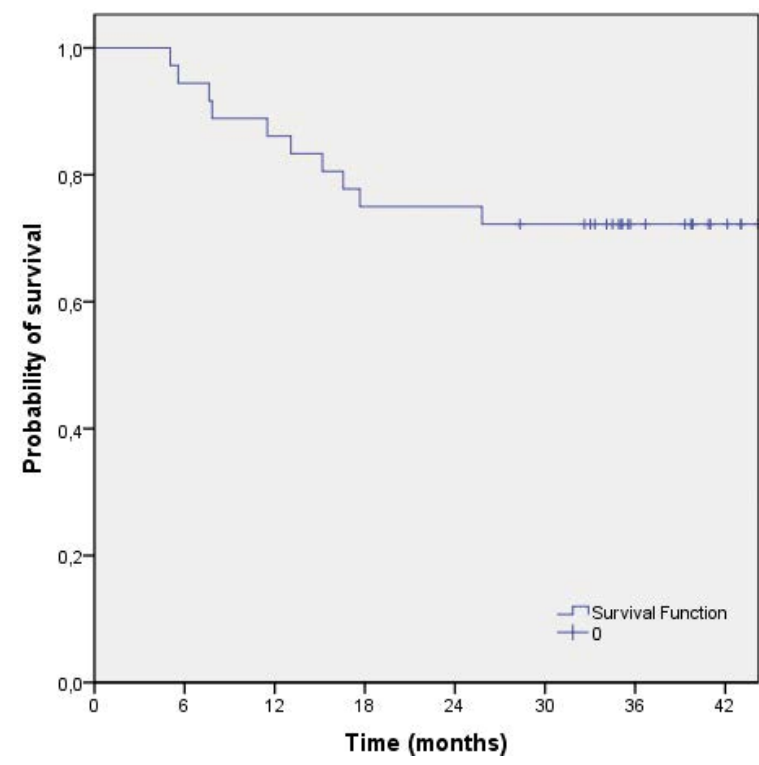

FIGURE 2. Disease-free survival of patients treated with preoperative capecitabine-base chemotherapy and cetuximab $(n=36)$.

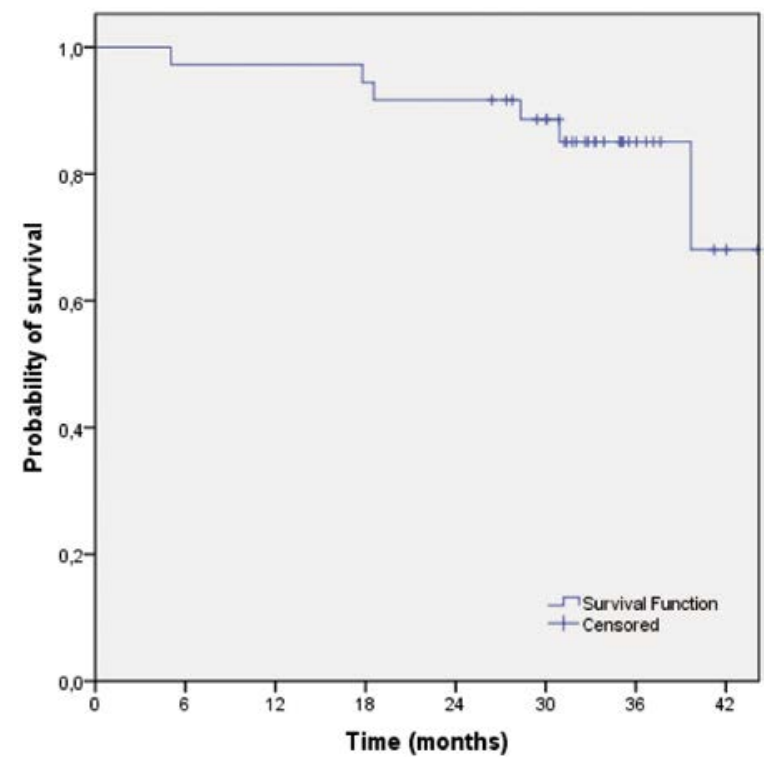

FIGURE 3. Overall survival of patients treated with preoperative capecitabine-base chemotherapy and cetuximab $(n=36)$.

CRT for LARC ${ }^{1}$, ways to improve the efficacy of preoperative CRT regimens have been investigated. In the study conducted by the German Rectal Cancer Study Group, the preoperative treatment comprised 5 Gy (in fractions of 1.8 Gy per day, five days per week) and fluorouracil $\left(1000 \mathrm{mg} / \mathrm{m}^{2} /\right.$ day 120-hour continuous infusion in the first and fifth weeks of radiotherapy). Four five-day cycles of fluorouracil $\left(500 \mathrm{mg} / \mathrm{m}^{2}\right)$ were administered one month after surgery. By five years, $6 \%$ of patients receiving preoperative therapy had relapsed. 
TABLE 1. Univariate analysis of disease-free survival according to patient, disease and treatment characteristics

\begin{tabular}{|c|c|c|c|}
\hline Gender & 0.010 & 1 & 0.919 \\
\hline Tumor location in the rectum (lower, middle or upper third) & 5.396 & 2 & 0.067 \\
\hline Type of surgery (abdominoperineal amputation or sphincter-sparing) & 2.311 & 2 & 0.315 \\
\hline $\begin{array}{l}\text { Dworak regression grade } \\
\qquad \begin{array}{l}1-4 \\
3-4 \text { vs } 0-2\end{array}\end{array}$ & $\begin{array}{l}7.966 \\
0.254\end{array}$ & $\begin{array}{l}4 \\
1\end{array}$ & $\begin{array}{l}0.093 \\
0.614\end{array}$ \\
\hline Tumour KRAS mutation status & 1.903 & 2 & 0.386 \\
\hline
\end{tabular}

$*$ Log-rank test. Number of patients $=36$.

In an earlier study from our group, we reported on the use of a capecitabine-based preoperative CRT regimen without cetuximab. ${ }^{3}$ The study used the same preoperative CRT regimen as that described in the present study but followed by four, rather than three, cycles of post-operative fluorouracil chemotherapy. Among 56 patients completing $\mathrm{CRT}$ and surgery, 55 had R0 resection. The pCR rate of $9.1 \%$ was similar to that reported in our current study with cetuximab and the five-year local control rate was $87.4 \%$ (95\% CI 75.0-99.8\%).

The original German Rectal Cancer Study Group trial reported a five-year overall survival rate of $76 \%$ for the use of preoperative CRT in LARC. ${ }^{1}$ In a recently reported randomized phase II study, the use of a dual chemotherapy combination of capecitabine and oxaliplatin in CRT followed by surgery and four cycles of post-operative capecitabine/oxaliplatin was reported to be associated with a three-year overall survival rate of $90 \%{ }^{23}$, although the acute grade $3 / 4$ toxicity associated with this regimen was fairly high: during CRT, $29 \%$ of patients experienced grade $3 / 4$ toxicity, mainly diarrhea. $^{24}$ The three-year overall survival results reported in our current study was $68.1 \%$, which may in part reflect the single-agent composition of the chemotherapy component of the CRT regimen. In a phase II study in 40 patients, the addition of irinotecan to capecitabine and cetuximab in the preoperative CRT regimen was associated with a three-year overall survival rate of $94.7 \%{ }^{17}$

It is now well established that patients with mCRC who have tumour KRAS mutations are unlikely to benefit from the addition of cetuximab to standard chemotherapy. ${ }^{25,26}$ However, in rectal cancer, the influence of KRAS mutation status on tumour response in the presence of radiation is unclear. Data from a number of studies have suggested both no association ${ }^{13,14,16}$ and a negative association $^{27}$ between the presence of tumour KRAS mutations and tumour regression and/or survival in patients with rectal cancer receiving preoperative CRT. In our study, no association between tumour KRAS mutation status and survival following preoperative cetuximab/capecitabine-based CRT could be found. Our findings support those from another analysis of data from 57 patients receiving CRT containing cetuximab plus irinotecan and capecitabine, in which tumour KRAS mutation status did not appear to influence tumour regression or disease-free survival. ${ }^{15}$ However, in a pooled analysis of data from 130 patients taking part in a number of phase II trials, including our own study, the presence of tumour KRAS mutations was significantly associated with pathologic nonresponse. ${ }^{21}$ It is possible, therefore, that tumour KRAS mutation status may be important in predicting outcome to cetuximab in this setting, but this would require larger scale analyses. Another study demonstrated that it was not KRAS mutation status that predicted achievement of a $\mathrm{PCR}$ following cetuximab-based neoadjuvant CRT, but rather the presence of $E G F A+61 G$ gene polymorphism, independent of KRAS mutation status. ${ }^{22}$ These data warrant additional investigation.

Our current study also reported relapse- and disease-free survival to be independent of gender, age, tumour location within the rectum, type of surgery, pathological $\mathrm{T}$ or $\mathrm{N}$ status and TRG, although, as for the KRAS analysis, the numbers of patients involved in the analysis are small. In a previous study, there was indication from a univariate 
analysis that post-operative parameters, including pathologic $\mathrm{T}$ or $\mathrm{N}$ status and TRG, were associated with disease-free survival. ${ }^{28}$ In multivariate analysis, however, only the association between $\mathrm{pN}$ and TRG and disease-free survival remained.

In conclusion, our study demonstrates that the addition of cetuximab to capecitabine-based preoperative CRT is feasible and is associated with an impressive three-year local control rate of $97 \%$. The use of tumour KRAS mutation status as a biomarker to identify which patients are most likely to respond to cetuximab-based CRT requires further, larger scale investigation.

\section{Acknowledgements}

The authors declare that they have no conflict of interests. Merck KGaA provided support as study drug (cetuximab) donation. Merck KGaA has reviewed the publication. The views and opinions described in the publication do not necessarily reflect those of Merck KGaA

\section{References}

1. Sauer R, Becker H, Hohenberger W, Rodel C, Wittekind C, Fietkau R, et al. Preoperative versus postoperative chemoradiotherapy for rectal cancer. $N$ Engl J Med 2004; 351: 1731-40.

2. Conde $\mathrm{S}$, Borrego $\mathrm{M}$, Teixeira $\mathrm{T}$, Teixeira $\mathrm{R}$, Corbal $\mathrm{M}$, Sá $\mathrm{A}$, et al. Impact of neoadjuvant chemoradiation on pathologic response and survival of patients with locally advanced rectal cancer. Rep Pract Oncol Radiother 2010; 15: 51-9.

3. Velenik V, Oblak I, Anderluh F. Long-term results from a randomized phase II trial of neoadjuvant combined-modality therapy for locally advanced rectal cancer. Radiat Oncol 2010; 5: 88.

4. Milas L, Mason K, Hunter N, Petersen S, Yamakawa M, Ang K, et al. In vivo enhancement of tumor radioresponse by $\mathrm{C} 225$ antiepidermal growth factor receptor antibody. Clin Cancer Res 2000; 6: 701-8.

5. Nasu S, Ang KK, Fan Z, Milas L. C225 antiepidermal growth factor receptor antibody enhances tumor radiocurability. Int J Radiat Oncol Biol Phys 2001; 51: 474-7.

6. Bonner JA, Harari PM, Giralt J, Azarnia N, Shin DM, Cohen RB, et al. Radiotherapy plus cetuximab for squamous-cell carcinoma of the head and neck. N Engl J Med 2006; 354: 567-78.

7. Bonner JA, Harari PM, Giralt J, Cohen RB, Jones CU, Sur RK, et al. Radiotherapy plus cetuximab for locoregionally advanced head and neck cancer: 5-year survival data from a phase 3 randomised trial, and relation between cetuximab-induced rash and survival. Lancet Oncol 2010; 11: 21-8.

8. Velenik V, Ocvirk J, Oblak I, Anderluh F. A phase II study of cetuximab, capecitabine and radiotherapy in neoadjuvant treatment of patients with locally advanced resectable rectal cancer. Eur J Surg Oncol 2011; 36: 244-50.

9. Zhou Y, Boardman LA, Miller RC. Genetic testing for young-onset colorectal cancer: case report and evidence-based clinical guidelines. Radiol Oncol 2010; 44: 57-61.

10. Lewander A, Gao J, Adell G, Zhang H, Sun XF. Expression of NF-kappa B p65 phosphorylated at serine- 536 in rectal cancer with or without preoperative radiotherapy. Radiol Oncol 2011; 45: 279-84.
11. Bokemeyer C, Bondarenko I, Hartmann JT, de Braud F, Schuch G, Zubel A, et al. Efficacy according to biomarker status of cetuximab plus FOLFOX-4 as first-line treatment for metastatic colorectal cancer: the OPUS study. Ann Oncol 2011; 22: 1535-46.

12. Van Cutsem E, Kohne CH, Lang I, Folprecht G, Nowacki MP, Cascinu S, et al. Cetuximab plus irinotecan, fluorouracil, and leucovorin as first-line treatment for metastatic colorectal cancer: updated analysis of overall survival according to tumor KRAS and BRAF mutation status. J Clin Oncol 2011; 29: 2011-9.

13. Bengala C, Bettelli S, Bertolini F, Sartori G, Fontana A, Malavasi N, et al Prognostic role of EGFR gene copy number and KRAS mutation in patients with locally advanced rectal cancer treated with preoperative chemoradiotherapy. Br J Cancer 2010; 103: 1019-24.

14. Davies JM, Trembath D, Deal AM, Funkhouser WK, Calvo BF, Finnegan T, et al. Phospho-ERK and AKT status, but not KRAS mutation status, are associated with outcomes in rectal cancer treated with chemoradiotherapy. Radiat Oncol 2011; 6: 114

15. Erben $P$, Strobel $P$, Horisberger K, Popa J, Bohn B, Hanfstein B, et al. KRAS and BRAF mutations and PTEN expression do not predict efficacy of cetuximabbased chemoradiotherapy in locally advanced rectal cancer. Int J Radiat Oncol Biol Phys 2011; 81: 1032-8.

16. Gaedcke J, Grade M, Jung K, Schirmer M, Jo P, Obermeyer C, et al. KRAS and BRAF mutations in patients with rectal cancer treated with preoperative chemoradiotherapy. Radiother Oncol 2010; 94: 76-81.

17. Kim SY, Hong YS, Kim DY, Kim TW, Kim JH, Im SA, et al. Preoperative chemoradiation with cetuximab, irinotecan, and capecitabine in patients with locally advanced resectable rectal cancer: a multicenter Phase II study. Int $J$ Radiat Oncol Biol Phys 2011; 81: 677-83.

18. Velenik V. Post-treatment surveillance in colorectal cancer. Radiol Oncol 2010; 44: 135-41.

19. Dworak O, Keilholz L, Hoffmann A. Pathological features of rectal cancer after preoperative radiochemotherapy. Int J Colorectal Dis 1997; 12: 19-23.

20. Mihaylova I, Parvanova V, Velikova C, Kurteva G, Ivanova D. Degree of tumor regression after preoperative chemo-radiotherapy in locally advanced rectal cancer - Preliminary results. Rep Pract Oncol Radiother 2011; 16: 237-42.

21. Grimminger PP, Danenberg P, Dellas K, Arnold D, Rodel C, Machiels JP, et al. Biomarkers for cetuximab-based neoadjuvant radiochemotherapy in locally advanced rectal cancer. Clin Cancer Res 2011; 17: 3469-77.

22. Hu-Lieskovan S, Vallbohmer D, Zhang W, Yang D, Pohl A, Labonte MJ, et al. EGF61 polymorphism predicts complete pathologic response to cetuximabbased chemoradiation independent of KRAS status in locally advanced rectal cancer patients. Clin Cancer Res 2011; 17: 5161-9.

23. Fernández-Martos $C$, Pericay C, Salud A, Massuti B, Alonso V, Safont $M$, et al. Three-year outcomes of GCR-3: a phase II randomized trial comparing conventional preoperative chemoradiation (CRT) followed by surgery and postoperative adjuvant chemotherapy (CT) with induction CT followed by CRT and surgery in locally advanced rectal cancer. [Abstract]. J Clin Oncol 2011; 29(15S): Abstract 3552

24. Fernández-Martos C, Pericay C, Aparicio J, Salud A, Safont M, Massuti B, et al. Phase II, randomized study of concomitant chemoradiotherapy followed by surgery and adjuvant capecitabine plus oxaliplatin (CAPOX) compared with induction CAPOX followed by concomitant chemoradiotherapy and surgery in magnetic resonance imaging-defined, locally advanced rectal cancer: Grupo Cáncer de Recto 3 study. J Clin Oncol 2010; 28: 859-65.

25. Bokemeyer C, Bondarenko I, Makhson A, Hartmann JT, Aparicio J, de Braud $\mathrm{F}$, et al. Fluorouracil, leucovorin, and oxaliplatin with and without cetuximab in the first-line treatment of metastatic colorectal cancer. J Clin Oncol 2009; 27: 663-71.

26. Van Cutsem E, Kohne CH, Hitre E, Zaluski J, Chang Chien CR, Makhson A, et al. Cetuximab and chemotherapy as initial treatment for metastatic colorectal cancer. N Engl J Med 2009; 360: 1408-17.

27. Garcia-Aguilar J, Chen Z, Smith DD, Li W, Madoff RD, Cataldo P, et al. Identification of a biomarker profile associated with resistance to neoadjuvant chemoradiation therapy in rectal cancer. Ann Surg 2011; 254: 486-93.

28. Vecchio FM, Valentini V, Minsky BD, Padula GD, Venkatraman ES, Balducci $M$, et al. The relationship of pathologic tumor regression grade (TRG) and outcomes after preoperative therapy in rectal cancer. Int J Radiat Oncol Biol Phys 2005; 62: 752-60. 